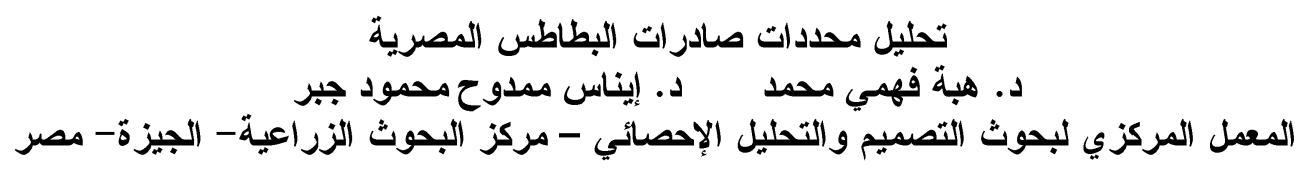

يعتبر القطاع الزراعي من القطاعات الهامة في جمهورية مصر العربية حيث أنه يساهم

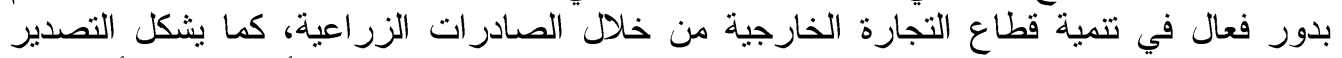

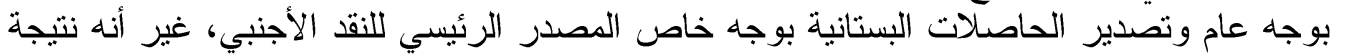

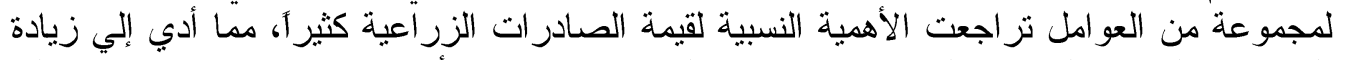

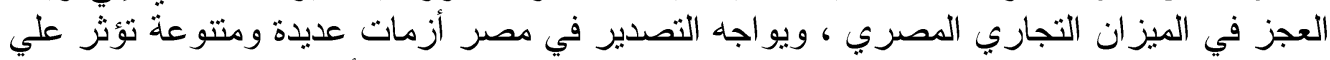

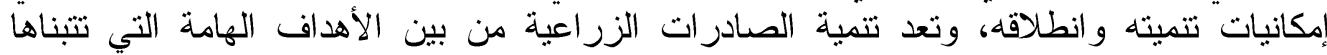
استر اتيجيات التنمية الزر اعية.

وتعتبر البطاطس من محاصيل الخضر ذاعن التهات الأهمية الغذائية الكبيرة حيث تعد الغذاء

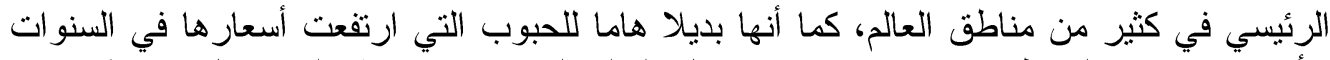

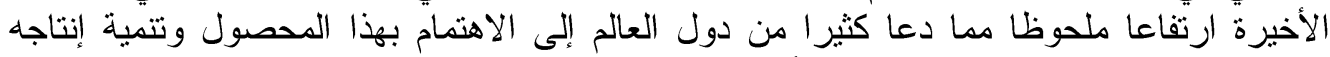

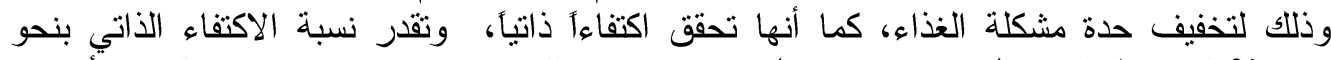

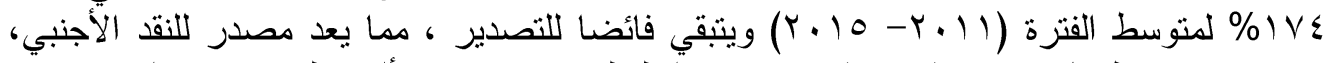

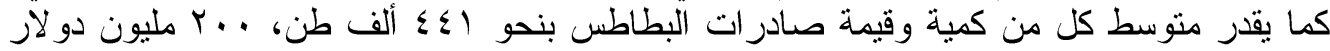

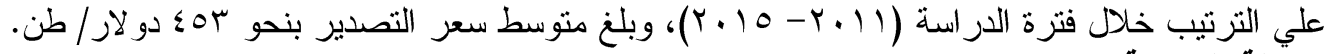

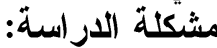

يؤدي تذبذب صادرات البطاطس المصرية المصدرة إلي الأسواق العالمية إلي عدم الطيات

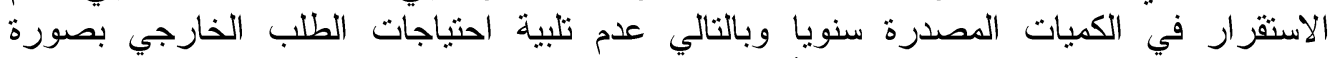

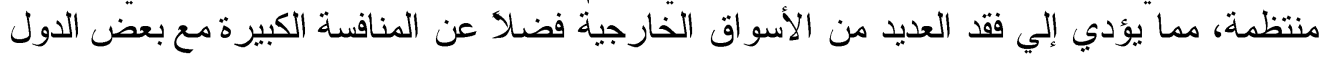

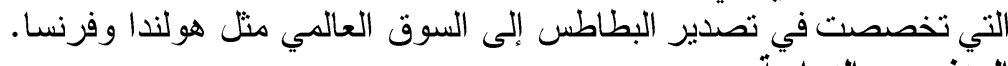
يستهدف البحث إيراز أهم المؤشرات التصديرية للبطاطس المصرية وتقدير معدل الاكتفاء البطاء

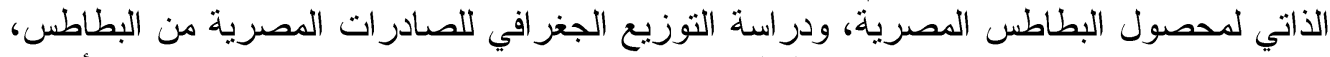

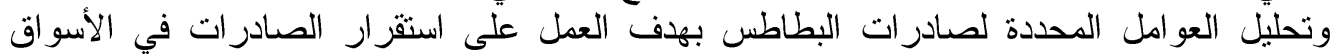

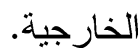
الطريقة البحثية ومصادر البيانات:

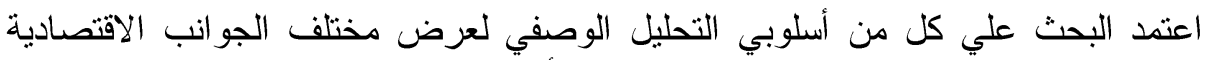

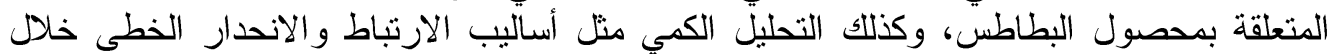

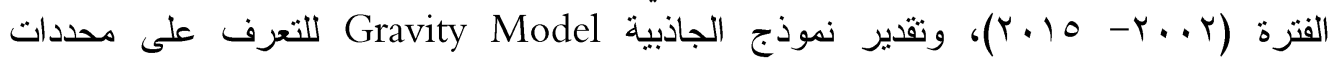

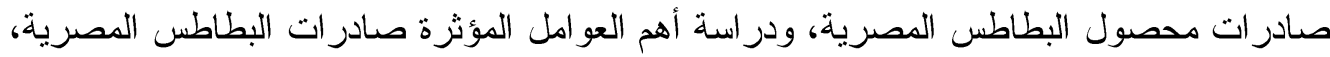

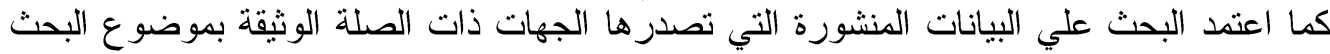

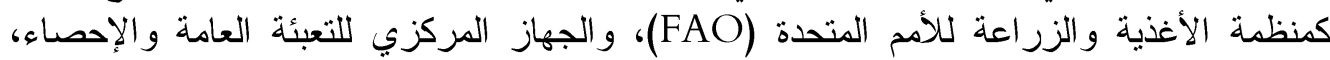

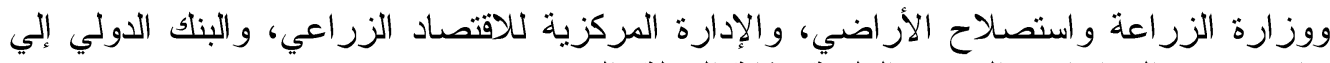

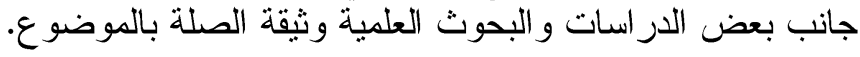

Fayoum J. Agric. Res. \& Dev., Vol. 32, No.1, January, 2018 


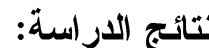 \\ أولاً: تطور المؤشر ات التصديرية البطاطس المصرية:

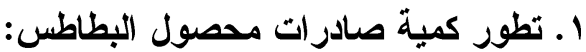

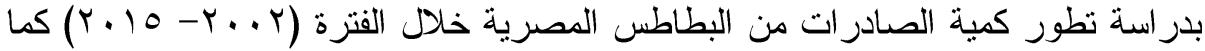

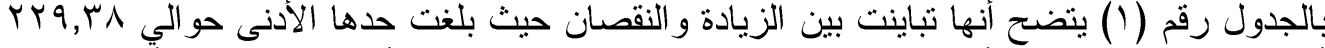

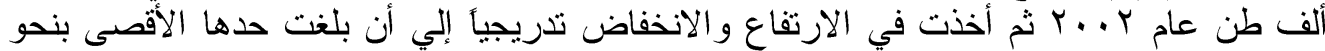
Tـ.

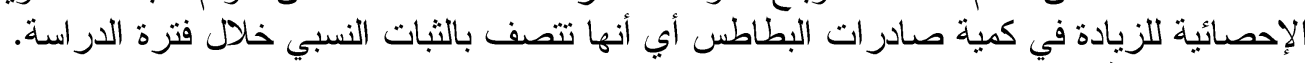

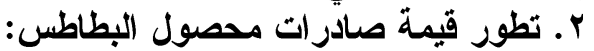

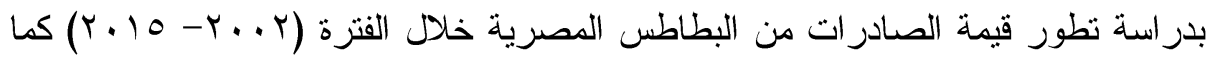

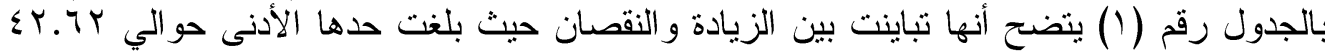

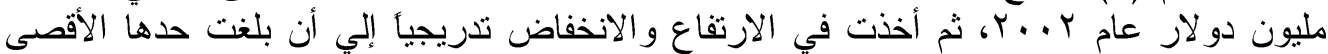

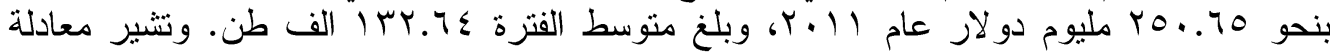

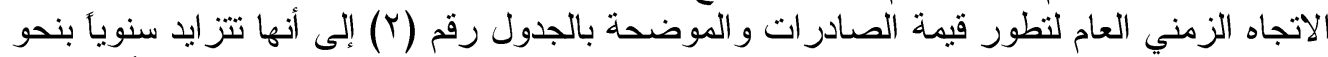

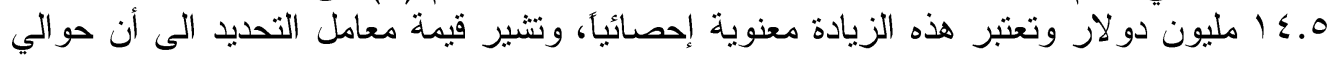

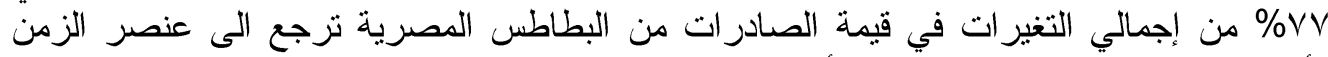

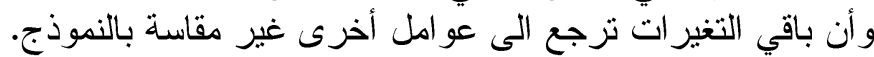

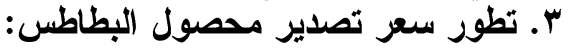

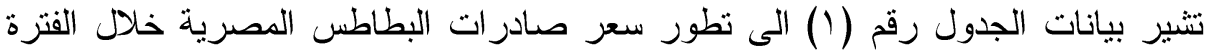

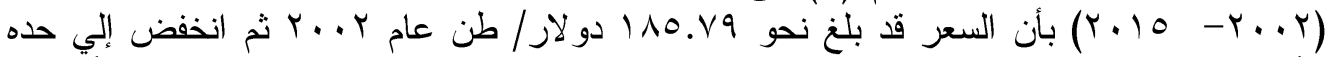

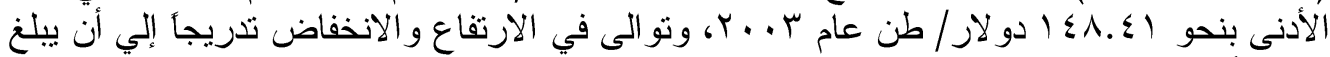

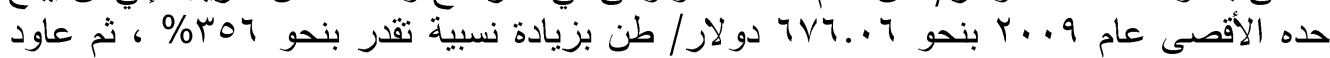

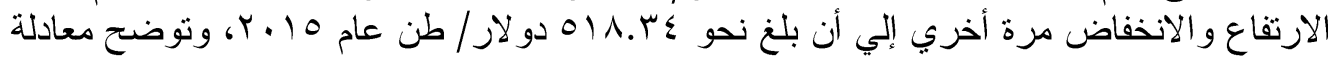

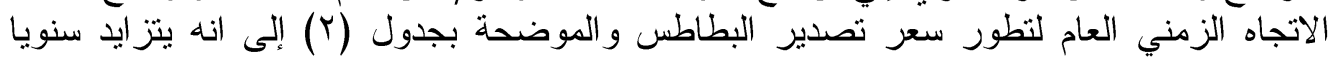

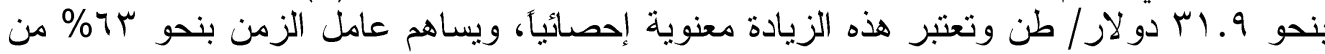

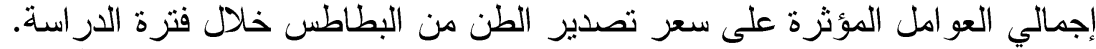

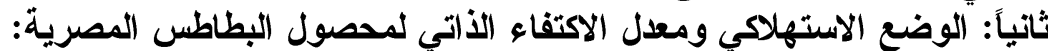

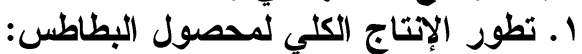

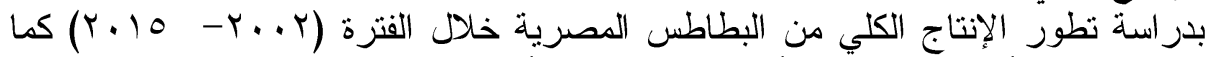

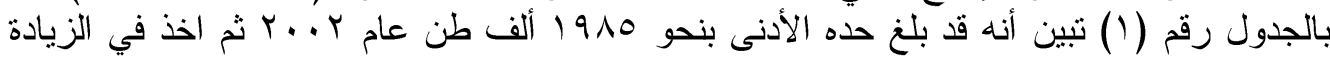

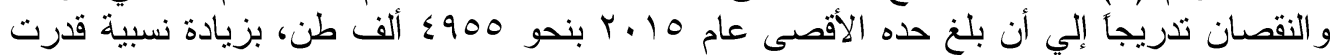

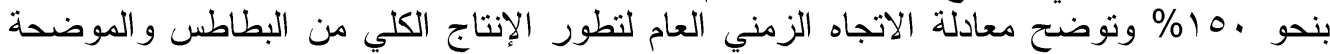

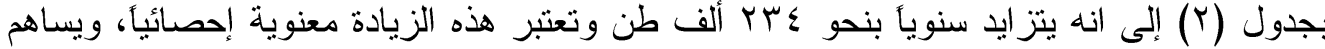

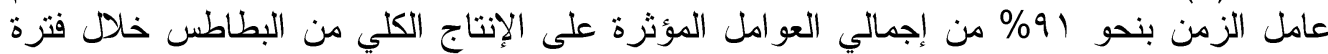

r. تطور الاستهلالك الكلي لمحصول البطاطس:

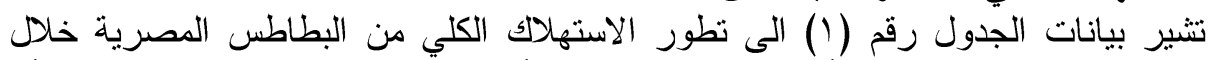

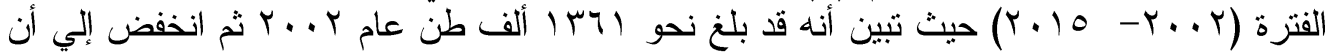

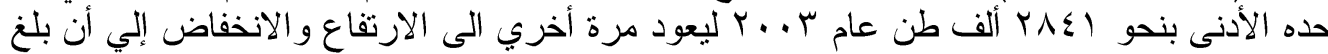

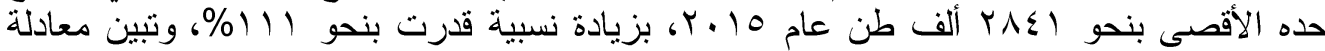

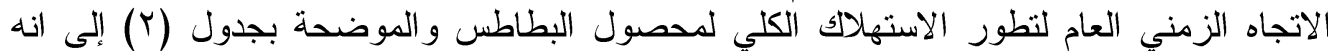

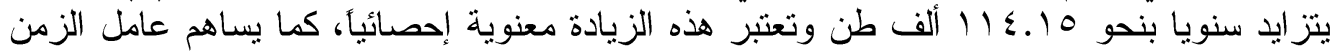

Fayoum J. Agric. Res. \& Dev., Vol. 32, No.1, January, 2018 
ґ

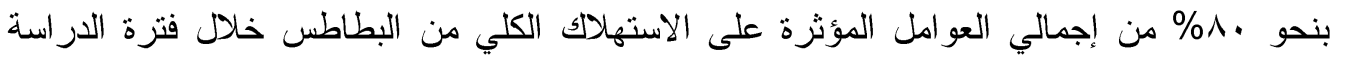

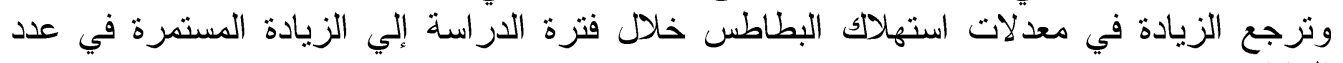

r. تطور معدل الاكتفاء الأتي لمحصول البطاطس:

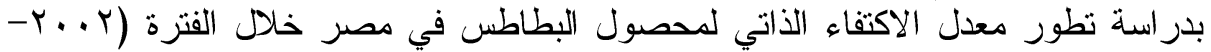

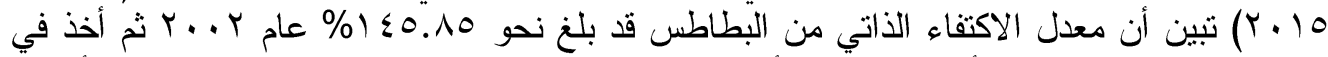

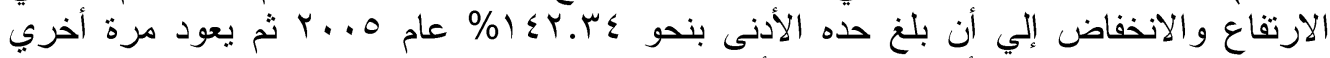

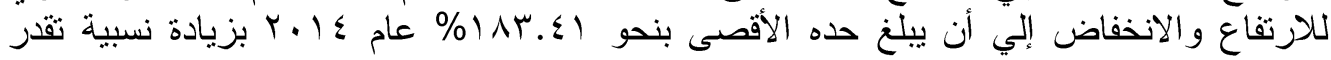

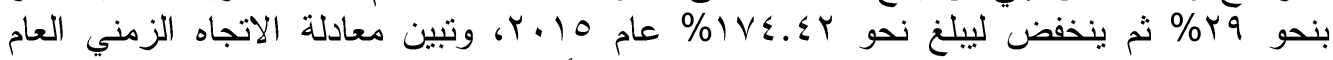

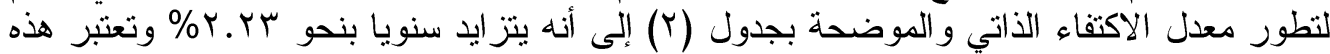

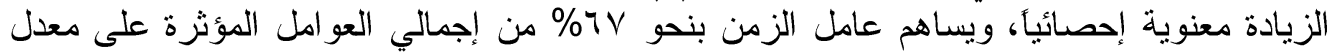
الاكتفاء الذاتي من البطاطس خلادل فئل فترة الدر استة.

\begin{tabular}{|c|c|c|c|c|c|c|}
\hline الأتثاء & بالألف طن & بالألف طن & بالملادرات & بالدولار & كالألف طادرات & السنو ات \\
\hline $1 \leqslant 0.10$ & 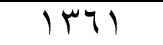 & 1910 & EY.TY & 110.19 & rYq.rA & $r \ldots r$ \\
\hline 101.54 & N & $r \cdot r q$ & $\leqslant \mu .9 V$ & $\mid \leqslant \Lambda . \leqslant 1$ & r 97. Q & $r \ldots r$ \\
\hline 170.0. & 1049 & $r 0 \leqslant V$ & TV.YT & IVT.KY & rol.01 & $r \ldots \varepsilon$ \\
\hline $1 \leqslant Y . \Psi \leqslant$ & TYYO & VITV & $V \vee . \leqslant 0$ & $19 V . \varepsilon \wedge$ & rar.l1 & $r \ldots o$ \\
\hline 109.04 & $1 \leqslant 0$. & אוسץ & ס. & $I V A$ & שT.ו & $Y . . T$ \\
\hline 101.ro & $I V \leqslant r$ & YVT. & $1.1 . .9$ & TVV.rV & 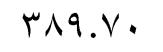 & $r . r v$ \\
\hline $101 . \leqslant V$ & rr. & ryog & 187.10 & $\leqslant \leqslant Y .70$ & rqv.q & $r \ldots \Lambda$ \\
\hline $10 \Lambda . \leqslant V$ & qTr.q & ryoq & $1 \leqslant 0 . \leqslant 1$ & $7 \vee 7 . .7$ & r $10 . .1$ & $r \ldots q$ \\
\hline IOV.r & Tr.q & צזוץ & $1 Y 9.07$ & ד & r91.07 & $r .1$. \\
\hline IVY.AT & rol. & 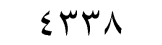 & Y0..10 & rqu.rr & r & $r .11$ \\
\hline $17 \Lambda . r v$ & rAYT & $\{\vee ० \wedge$ & IKV.ro & $\varepsilon \Lambda \varepsilon . Y_{0}$ & rTr.99 & $r .1 r$ \\
\hline $1 V \cdot . \leqslant 4$ & ro.r & ETTO & 119 & 219.04 & $\{0 . .0\}$ & $r .1 r$ \\
\hline$|\wedge \mu . \varepsilon|$ & rol $\varepsilon$ & $\{7\rceil)$ & $\langle 1 . . \leqslant 1$ & $\leqslant 9 \Lambda ., 1$ & Err.o. & $r+1 \varepsilon$ \\
\hline $\mid V \leqslant . \leqslant Y$ & rAミl & $\leqslant 900$ & TrT.T9 & $011 . r \leqslant$ & $\{\pi 1.00$ & $r .10$ \\
\hline 171.9. & YITV.OV & T $\leqslant \vee V . \wedge T$ & ITY.TE & roq.Y & "ч9.รᄉ & المتوسط \\
\hline
\end{tabular}

1- بيانات منظمة الزر اعة و الأغذية F.A.O قاعدة بيانات شبكة الانترنت.

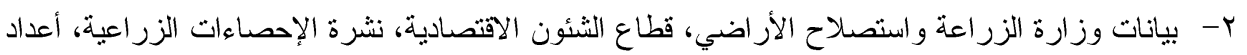

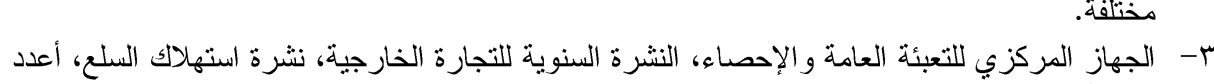

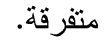

Fayoum J. Agric. Res. \& Dev., Vol. 32, No.1, January, 2018 
rq

جدول (Y): معادلات الاتجاه الزمني العام لتطور مؤشرات الصادرات والاكتفاء الأتي من البطاطس

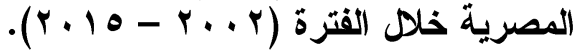

\begin{tabular}{|c|c|c|c|c|c|}
\hline المعنوية & $\mathbf{F}$ & $\mathbf{R}^{2}$ & $\mathbf{R}$ & المعادلة & البيان \\
\hline ** & E..Yo &..$V V$ &.$\wedge \wedge$ & $\hat{Y}_{i}=\underset{(1.24)^{-}}{24.01+} \underset{(6.34)^{* *}}{14.48} X_{i}$ & بآيمة الصادر اتيون دولار \\
\hline ** & r. & r. & .189 & $\hat{Y}_{i}=\underset{(2.13)^{*}}{126.07}+\underset{(4.48)^{* * *}}{31.09} X_{i}$ & سالدو الار / طنيز \\
\hline ** & 110.99 &. .91 & .990 & $\hat{Y}_{i}=\underset{(9.35)^{*}}{1727}+\underset{(10.77)^{* *}}{234} X_{i}$ & \\
\hline$* *$ & $\leqslant 9.0$ &.$\wedge$. & .9. & $\hat{Y}_{i}=\underset{(9.16)^{* * *}}{1271+}+\underset{(7.00)^{* * *}}{114.15} X_{i}$ & الاستهلأك الكلي طن \\
\hline$* *$ & YE.VT &. $.7 V$ &.$\Delta Y$ & $\hat{Y}_{i}=\underset{(38)^{* *}}{145.16}+\underset{(4.98)^{* *}}{2.23} X_{i}$ & معدل الاكتقاء \\
\hline
\end{tabular}

المصدر: جمعت وحسبت من بيانات الجدول (1)

i القيمة التقديرية للمتغير التابع في السنةi. .

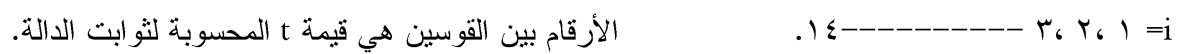

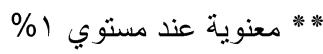

ثالثاً: نسبة مساهمة قيمة صادرات البطاطس المصرية في الصادرات الزراعية:

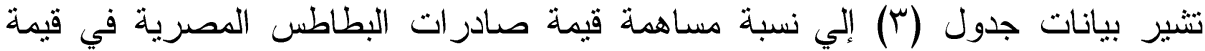

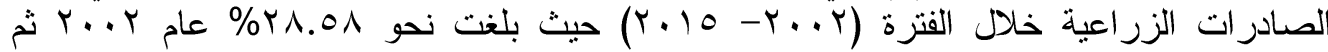

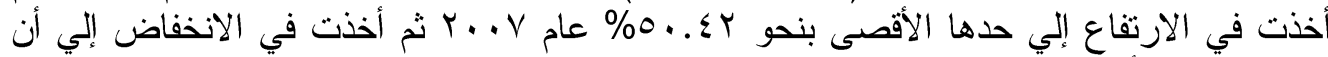

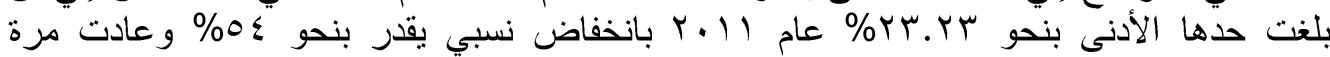

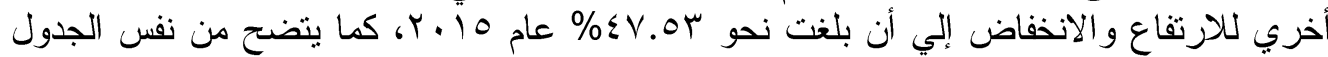

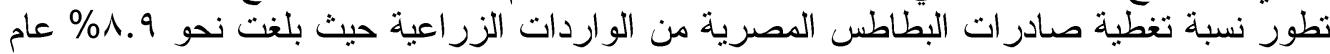

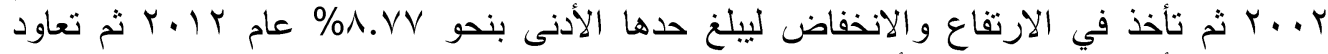

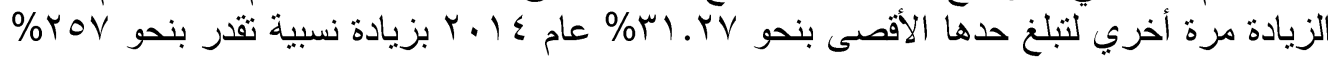

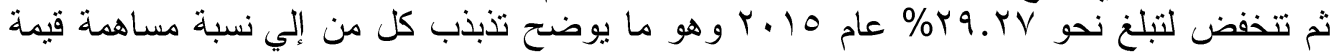

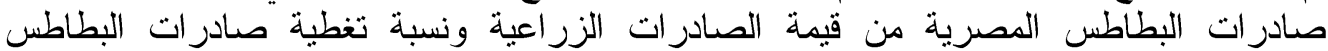

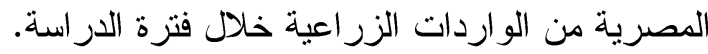

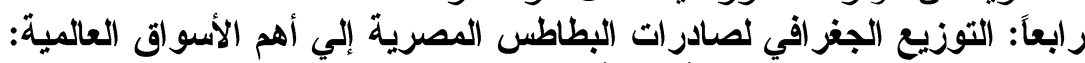

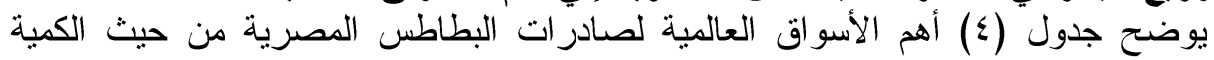

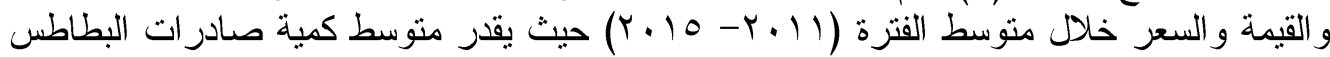

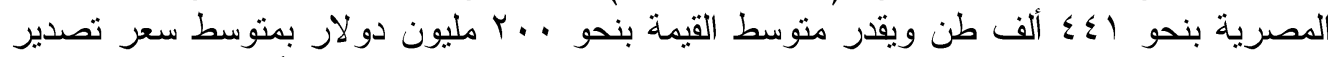

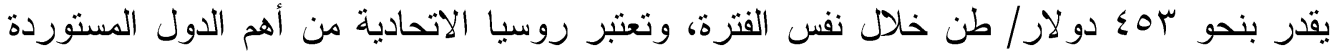

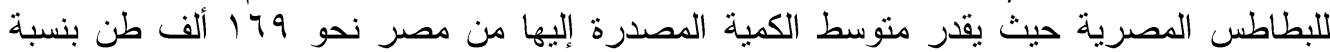

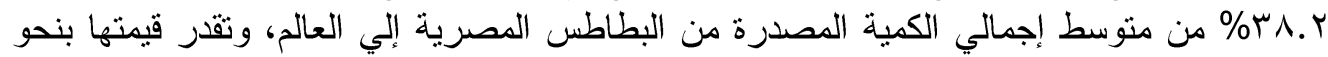

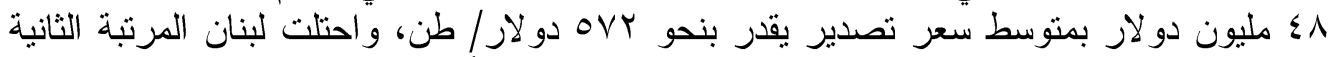

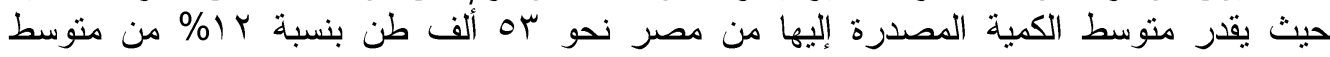

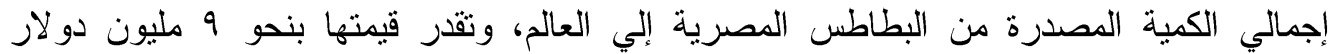
بمتوسط سعر تصدير يقدر بنحو و؟ب دولار/ طن، وتأتي ايطاليا في المرتبة الثالثة حيث يقدر

Fayoum J. Agric. Res. \& Dev., Vol. 32, No.1, January, 2018 
$\varepsilon$.

منوسط الكمية المصدرة إليها من مصر نحو سه ألف طن بنسبة r أ\% من متوسط إجمالي الكمية

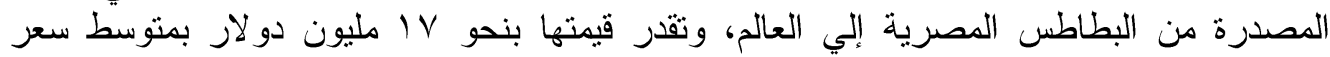

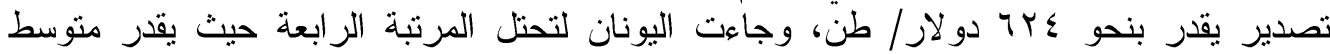

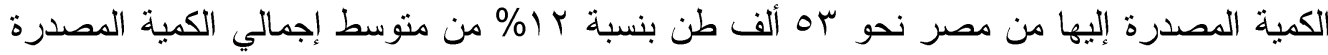

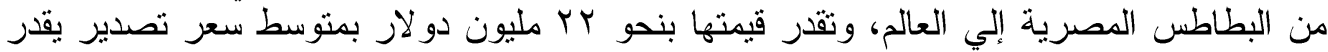

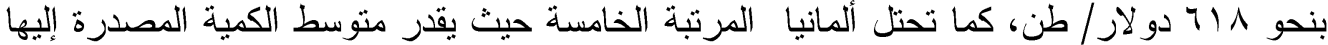
من مصر نحو .ب ألف طن بنسبة 9.7\% من متوسط إجمالي الكمية المصدرة من البطاطس المصرية إلي العالم وتقدر قيمتها بنحو 9 مليون دولار بمتوسط سعر تصدير يقدر بنحو

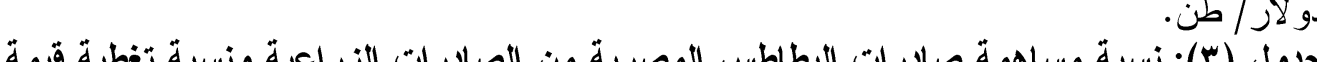

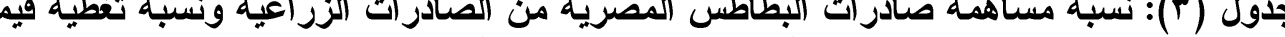

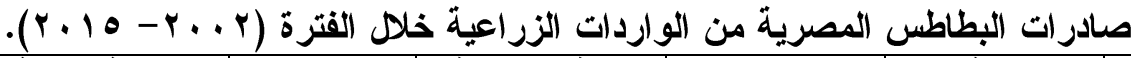

\begin{tabular}{|c|c|c|c|c|c|}
\hline 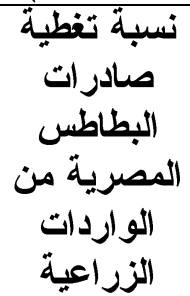 & 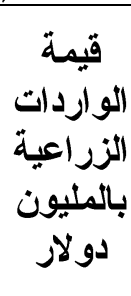 & قيمة قالبة صادر مساهمة & الصادرة الزيمة اعلية & البادية & السنو ات \\
\hline 1.9. & $\varepsilon \vee q$ & $r \wedge .0 \Lambda$ & $1 \leqslant 9$ & EY.YY & $r \ldots r$ \\
\hline 10.rV & rAᄉ & סן.سץ & سזן & $\varepsilon r .9 V$ & $r . . r$ \\
\hline$r \leqslant . \leqslant 0$ & rVo & rV.Vr & IVA & מז.VI & $r \ldots \varepsilon$ \\
\hline 11.09 & $\{1 V$ & $\varepsilon \wedge . \vee\urcorner$ & 109 & $V V . \leqslant 0$ & $r . .0$ \\
\hline 17.0. & & $\varepsilon \mu . \wedge$. & $1 \leqslant 9$ & To.ro & $Y . . q$ \\
\hline $17 . V Y$ & $T \leqslant V$ & $0 . . V Y$ & rIT & $1 \cdot 1.99$ & $r . . V$ \\
\hline 19.10 & $\Lambda \wedge \vee$ & $\leqslant 0 . v 9$ & MAo & $1 V 7.10$ & $r . \Lambda$ \\
\hline ס א.11 & Vqr & rV.10 & דry & $1 \leqslant 0 . \leqslant 1$ & $r . . q$ \\
\hline r. & 1 roq & سץ. & 001 & $1 Y 9.07$ & $r .1$. \\
\hline 11.11 & ITAE & $\leqslant 9.10$ & 01. & Yo. & Y.11 \\
\hline A. VV & $1 \leq 04$ & r^.VT & $\varepsilon \varepsilon \mu$ & ס & $r .1 r$ \\
\hline rY.VT & Ar. & $\varepsilon . V_{0}$ & 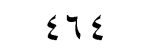 & 119 & $r .1 r$ \\
\hline T. IV & TVT & $\leqslant r . \leqslant q$ & $\varepsilon \wedge \varepsilon$ & $r_{1} \cdot . \leqslant 1$ & $Y .1 \varepsilon$ \\
\hline rq.VT & vor & EV.OH & $\varepsilon \vee 1$ & q.ד. & $r .10$ \\
\hline
\end{tabular}

تأتي انجلتر ا في المرتبة السادسة حيث يقدر متوسط الكمية المصدرة إليها من مصر نحو ه ألف طن بنسبة 7\% من متوسط إجمالي الكمية المصدرة من البطاطس المصرية إلي العالم، وتقدر قيمتها بنحو 11 مليون دولار بمتوسط سعر تصدير يقدر بنحو NVI دولار/ طن، وتحتل

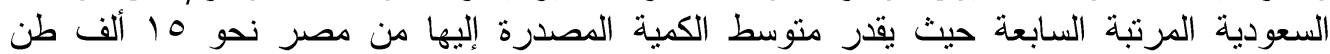
بنسبة ب\% من متوسط إجمالي الكمية المصدرة من البطاطس المصرية إلي العالم وتقدر قيمتها بنحو

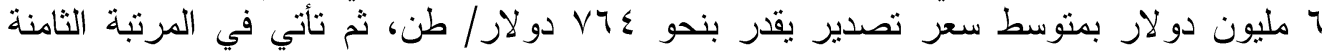

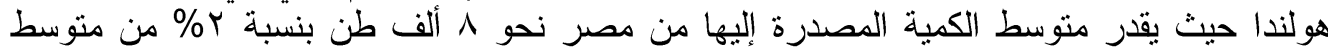

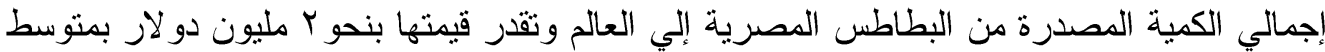

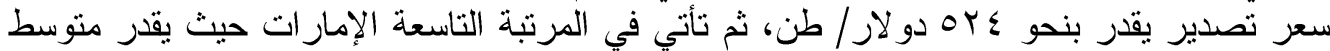

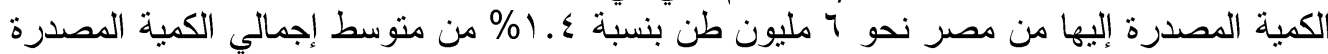

Fayoum J. Agric. Res. \& Dev., Vol. 32, No.1, January, 2018 
«

من البطاطس المصرية إلي العالم وتقدر قيمتها بنحو r مليون دولار بمتوسط سعر تصدير يقدر

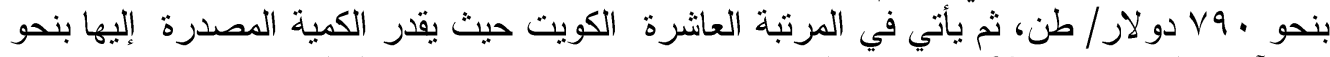

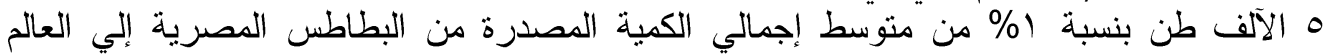

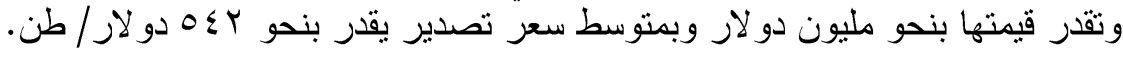

جدول(ع) التوزيع الجغرافي لصادرات البطاطس المصرية لأهم الأسواق العالمية خلا القترة $\cdot(r+10-r+11)$

\begin{tabular}{|c|c|c|c|c|c|}
\hline \multirow[b]{2}{*}{ تصدير الطن سبر } & \multirow{2}{*}{ متوسط قيمة } & \multicolumn{3}{|c|}{ متوسط الكمية المصدرة } & \multirow[b]{2}{*}{ الدولة } \\
\hline & & الترتيب & \% المتوسط جملة & بالألف طن & \\
\hline OVY & $\leqslant \wedge$ & 1 & rА. & 179 & روسيا الاتحادية \\
\hline$r \leqslant q$ & 9 & r & ir & or & لبنان \\
\hline E & iv & r & ir & r & ايطاليا \\
\hline $71 \wedge$ & rt & $\varepsilon$ & Ir & r & اليونان \\
\hline$O \wedge \Lambda$ & 9 & 0 & 7.9 & r. & ألمانيا \\
\hline AV & 11 & 1 & 7 & ro & انجلتر | \\
\hline VI & 7 & V & r & 10 & السعودية \\
\hline OY & r & $\wedge$ & r & $\wedge$ & هو لندا \\
\hline $\mathrm{vq}$. & r & 9 & $1 . \varepsilon$ & 7 & الإمار ات \\
\hline $0 \leqslant r$ & 1 & 1. & 1.1 & 0 & الكويت \\
\hline 7.9 & $\mathrm{~V}$ & - & $0 . \varepsilon$ & $r \varepsilon$ & أخري \\
\hline$\varepsilon \Delta r$ & r.. & & $1 \ldots$ & $\varepsilon \leqslant 1$ & متوسط الكمية \\
\hline
\end{tabular}

خامساً: مؤشر ات التجارة الخارجية لمحصول البطاطس

ا

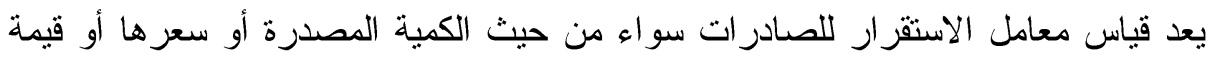

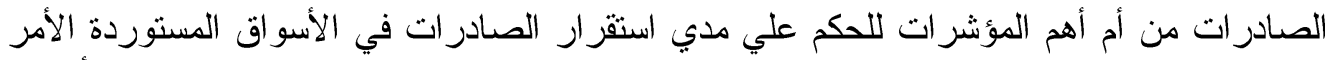

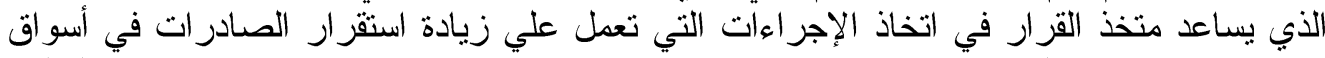

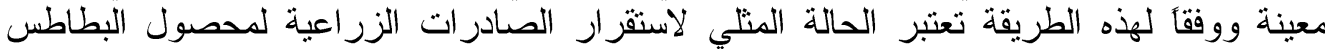

عندما يكون معامل الاستقرار مساوياً للصفر ويمكن حساب معامل الاستقرار من المعادلة التالية:

$$
S C=\left(\frac{Y_{i}-\hat{Y}_{i}}{\hat{Y}_{i}}\right) * 100
$$

= معامل الاستقرار = SC $i$ السنة

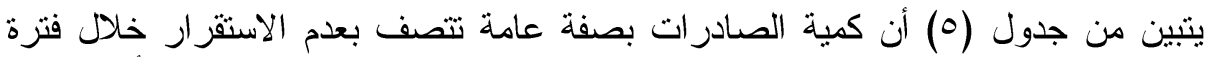

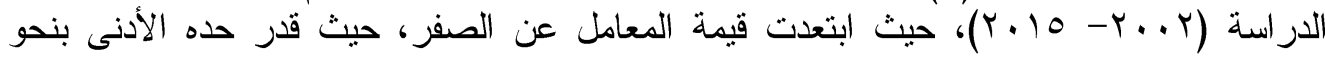

Fayoum J. Agric. Res. \& Dev., Vol. 32, No.1, January, 2018 


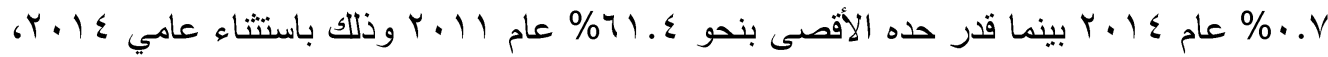

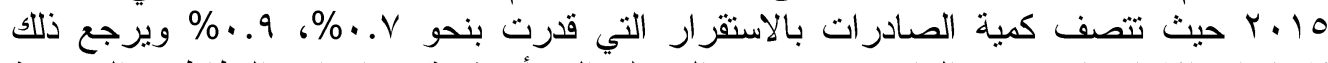

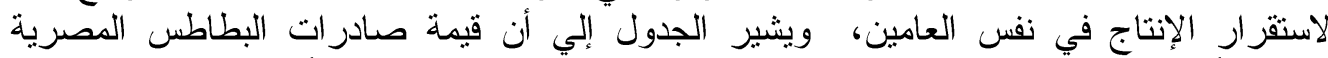

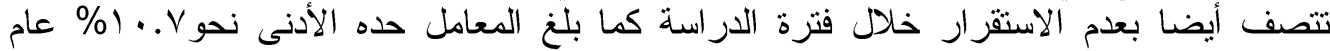

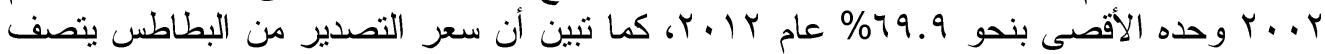

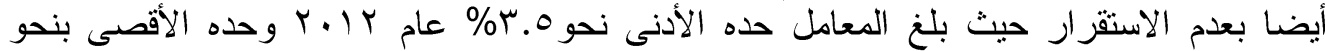

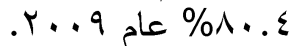

جدول (•): معامل عدم الاستقرار لكمية وقيمة وسعر تصدير البطاطس المصرية خلال الفترة . $(r \cdot 10-r . r)$

\begin{tabular}{|c|c|c|c|}
\hline \% الاستقرار لسعل عدم & \% لمعامل عدم الاستقرار & \% للكمبة المتقرامل عدرم & السنوات \\
\hline IA.r. & $1 . . v$. & $r \xi . \varepsilon,-$ & $r \ldots r$ \\
\hline YI.r.- & $\leqslant r . q .-$ & $0.0 .-$ & $r \ldots r$ \\
\hline $19.8 .-$ & $\leq 1 . \wedge .-$ & IY.9. & $r \ldots \varepsilon$ \\
\hline Y..1. - & $\leqslant q . \vee .-$ & IY.O. & r...o \\
\hline ب....- & 7ז...- & T.V. & $Y \ldots T$ \\
\hline $11 . r .-$ & Or.Y.- & $1 \ldots$ & $Y \ldots V$ \\
\hline YA.A. & $\Gamma \leqslant .7 .-$ & Q.Y. & $r \ldots \Lambda$ \\
\hline$\Lambda \cdot . \varepsilon$. & or.A.- & $\leqslant Y . T .-$ & $r \ldots q$ \\
\hline 7.9. & TY.T.- & YY.E.- & $r .1$. \\
\hline $1 \ldots$. & $r \varepsilon .9 .-$ & Tl.s. & $r .11$ \\
\hline r.o. & $79.9 .-$ & ro.1.- & $r .1 Y$ \\
\hline $10.9 .-$ & $09.1 .-$ & 1.0 & $r .1 T$ \\
\hline $7.1 .-$ & O^....- & $\therefore V-$ & Y.l $\leq$ \\
\hline$V \cdot V \cdot-$ & $01.0 .-$ & $\cdot .9-$ & $r .10$ \\
\hline
\end{tabular}

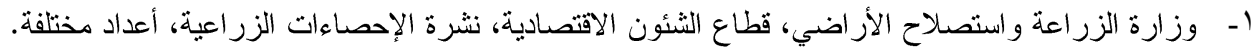

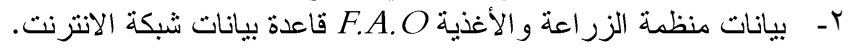

r. التنافسية السعرية للبطاطس المصرية: Price competitiveness

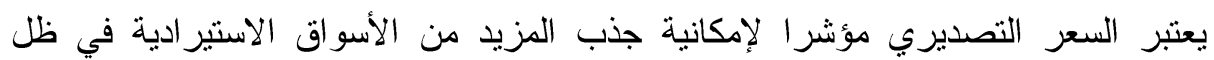

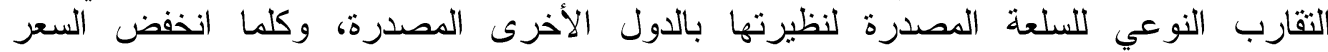

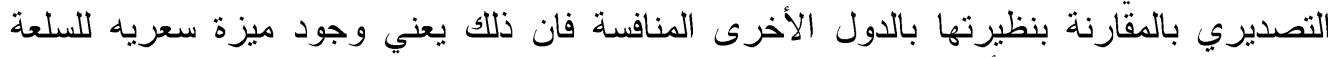

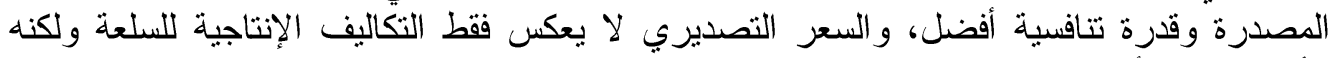

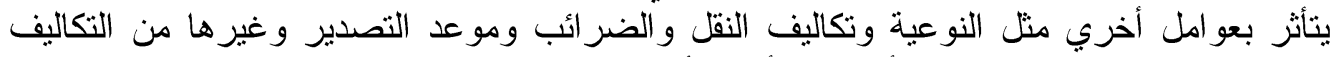

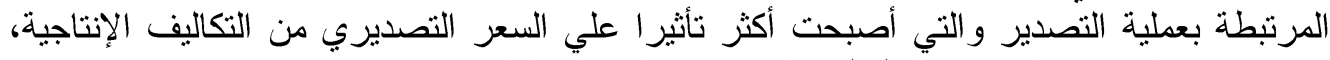
ويتم حساب التنافسية السعرية للبطاطس النس المصرية بالمعادلة التالية:

Fayoum J. Agric. Res. \& Dev., Vol. 32, No.1, January, 2018 


$$
\begin{aligned}
& P C=\frac{P E_{j i}}{P C_{j i}} \\
& \text { PC } \\
& i \text { متوسط سعر التصدير للسلعة j في السنة }
\end{aligned}
$$

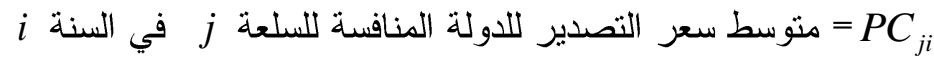

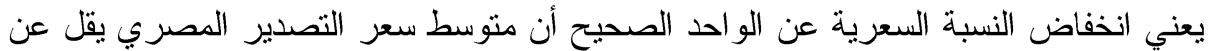

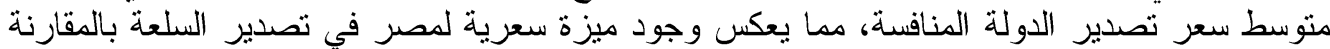
بالدول المنافسة و العكس صحيح.

يوضح جدول (؟) بتقدير قيمة التتافسية السعرية لسعر تصدير البطاطس المصرية بالنسبة لأهم

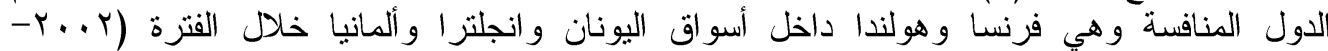

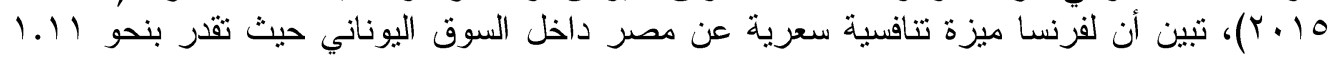

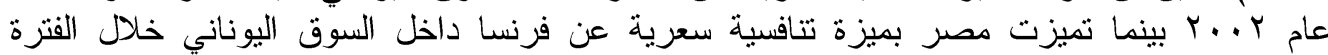

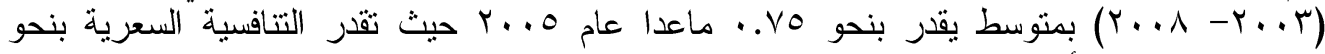

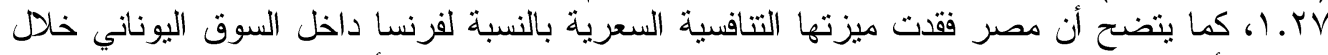

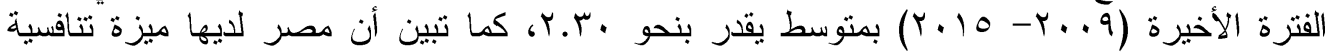

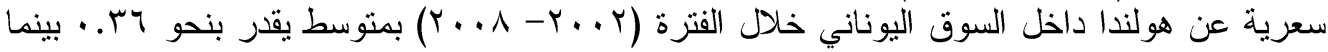

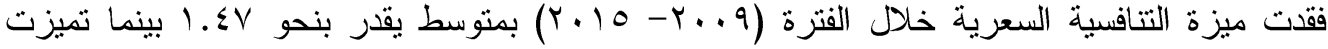

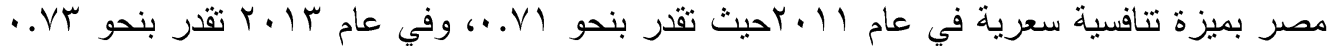

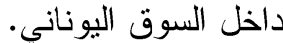

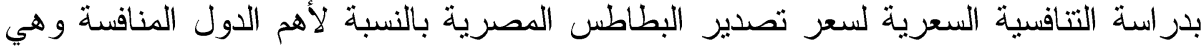

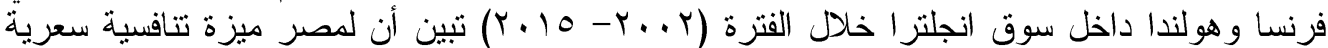

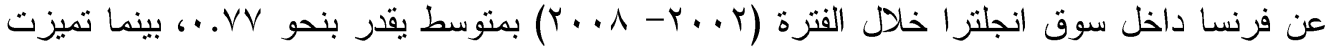

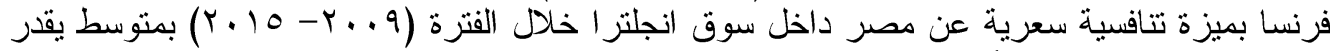

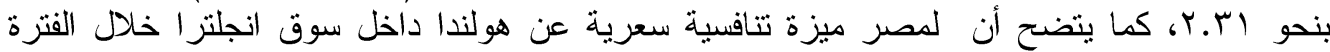

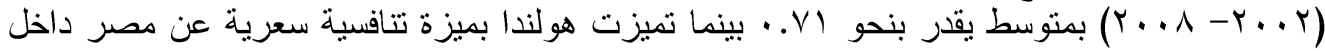

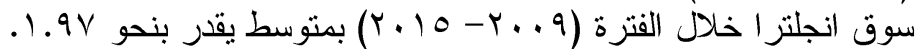

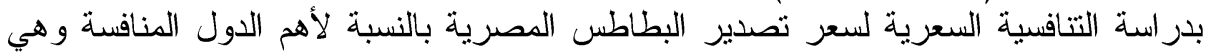

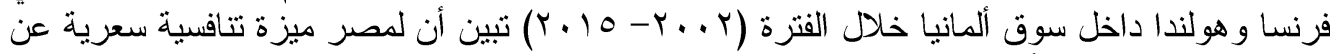

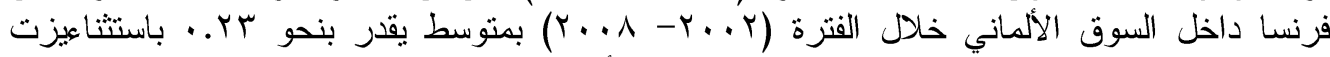

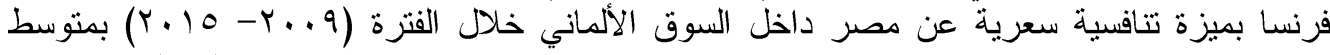

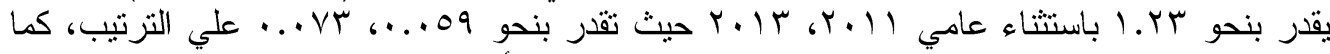

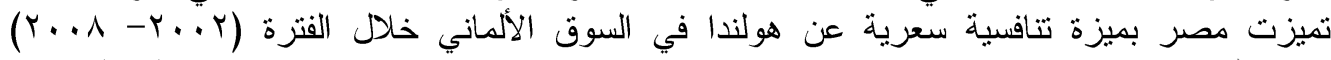

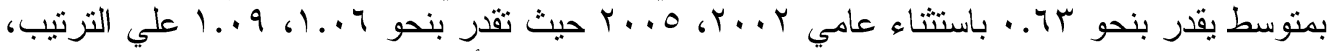

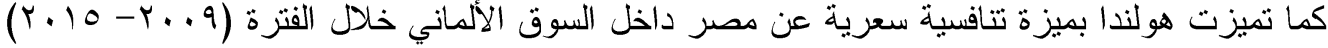

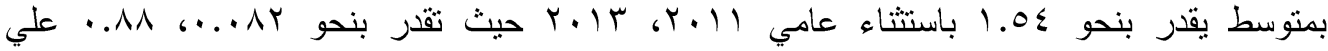

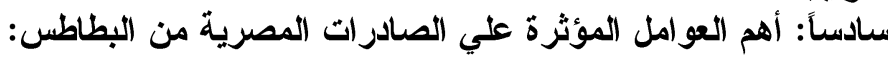

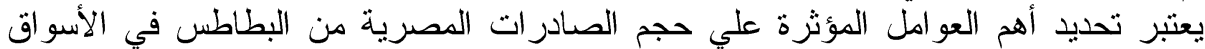

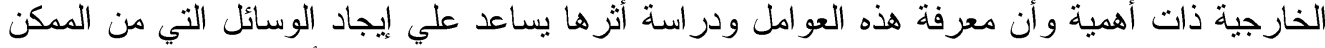

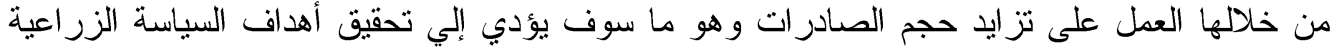

Fayoum J. Agric. Res. \& Dev., Vol. 32, No.1, January, 2018 
$\varepsilon \varepsilon$

المصرية التي يعتبر هدفها الأساسي زيادة الحصيلة التصديرية الزراعية وتتوعها وعدم الاعتماد علي ع عاي محاصيل محدة. جدول (†): تطور التنافسية السعرية لمحصول البطاطس المصرية في أسواق اليونان وانجلترا وألمانيا

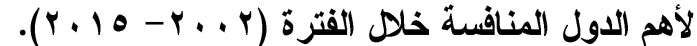

\begin{tabular}{|c|c|c|c|c|c|c|}
\hline \multicolumn{2}{|c|}{ سوق ألماتيا } & \multicolumn{2}{|c|}{ سوق اتجلترا } & \multicolumn{2}{|c|}{ سوق اليونان } & \multirow{2}{*}{ السنوات } \\
\hline هولندا & فرنسا & هولندا & فرنسا & هولندا & فرنسا & \\
\hline 1.97 & .7. & .991 &..$\vee \wedge$ &..$\leqslant \varepsilon$ & 1.11 & $r \ldots r$ \\
\hline .10 & .0. & $. V \leqslant$ &.$V Y$ & .149 &.$\vee \wedge \wedge$ & $r \ldots r$ \\
\hline .70 & ו &.$V$. & $. V \leqslant \leqslant$ & سז. &. .71 & $r \ldots \varepsilon$ \\
\hline 1.99 & $\because$ Vo &.$V V$ & • . & $\cdot . \leqslant \wedge$ & $1 . Y V$ & $r \ldots o$ \\
\hline$\cdot . \leqslant 9$ & 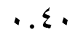 & 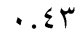 & .04 & . ז.. & $.0 T$ & $r \ldots r$ \\
\hline. $.0 \mathrm{~V}$ &..$\leqslant 7$ & $\cdot . \leqslant \wedge$ &.$V Y$ & דמ.. & $\cdot . \wedge$ & $r . r v$ \\
\hline .7 & $\cdot . \leqslant \Lambda$ & .9. &. .99 &.$\leqslant 0$ & $.9 \leq$ & $r \ldots \Lambda$ \\
\hline Y... $\Sigma$ & $1 . \leqslant 0$ & 1.71 & r.19 & س. & r. $\leqslant q$ & $r \ldots q$ \\
\hline $1 . \leqslant \varepsilon$ & $1 . .9$ & 1.10 & Y.. I & 1.11 & Y..A & $r .1$. \\
\hline.$\Delta r$ & .09 & $1.1 \mathrm{~V}$ & $1 . \leqslant V$ &.$v 1$ & 1.17 & $r .11$ \\
\hline $1 . V Y$ & 1.19 & 1.14 & Y..V & ז.7. & r.97 & $r .1 r$ \\
\hline..$\wedge \Lambda$ &.$V r$ & T.TY & T.VT & س & 1.1. & $r .1 r$ \\
\hline $1 . \leqslant Y$ & 1.10 & r. 79 & T.Y & $1.0 \leqslant$ & T.r. & $r .1 \varepsilon$ \\
\hline $1.0 \leqslant$ & $1 . Y V$ & Y.. $\varepsilon$ & Y. $\leqslant T$ & $1.7 \mathrm{~V}$ & $Y . \leqslant \Lambda$ & $r .10$ \\
\hline
\end{tabular}

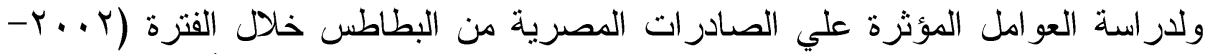
10

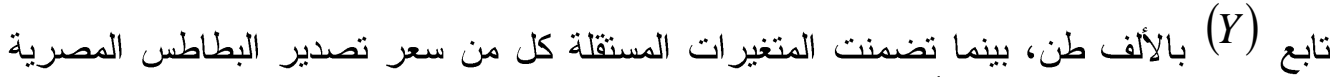

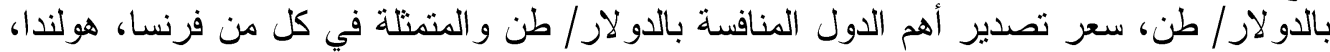

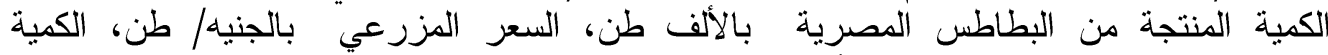

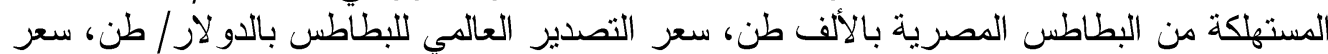

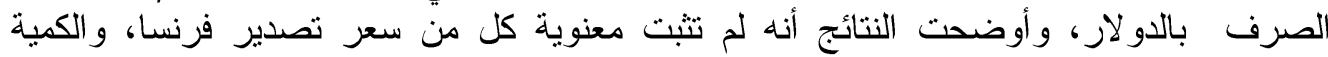

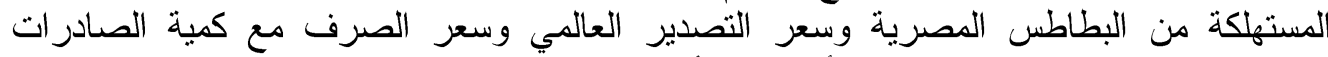

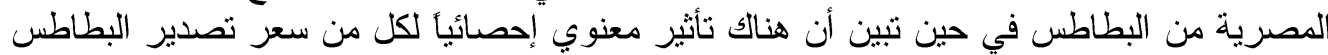

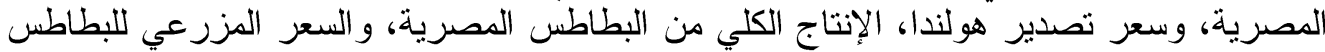

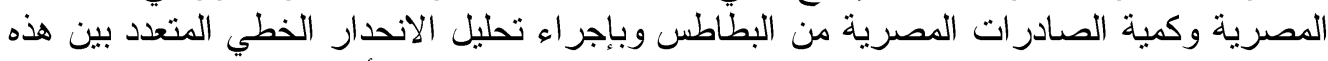

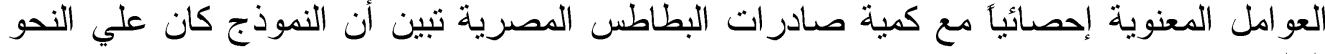

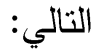

Fayoum J. Agric. Res. \& Dev., Vol. 32, No.1, January, 2018 
$\varepsilon \theta$

$$
\begin{aligned}
& \hat{Y}_{i}=\underset{(0.40)^{-}}{26.53}-\underset{(-6.24)^{* * *}}{1.36} X_{1}+\underset{(4.91)^{* * *}}{1.45} X_{2}+\underset{(5.08)^{* * *}}{0.16} X_{3}-\underset{(-2.26)^{*}}{0.29} X_{4} \\
& R^{2}=0.84 \quad \bar{R}^{2}=0.77 \\
& F=(11.87)^{\text {** }} \\
& R=0.92 \\
& \text { = } \hat{Y}_{I}
\end{aligned}
$$$$
\text { = } X_{1}
$$$$
\text { = سعر تصدير هولندا بالدو لار / طن. }
$$$$
\text { = } X_{3}
$$$$
\text { X } X_{4}
$$

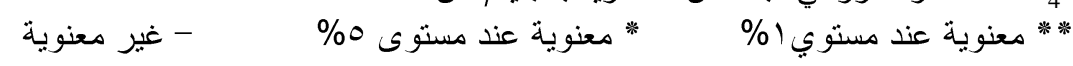

وأوضحت النتائج أن هناك تأثير معنوي إحصائياً لهذه العوامل السابقة على كمية

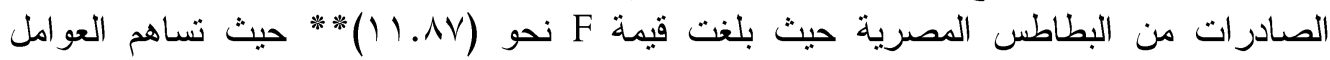

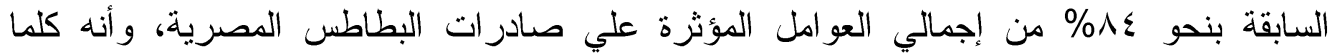

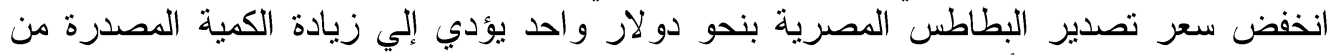

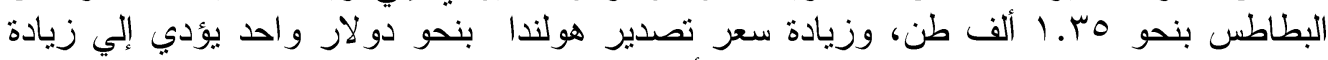
الكمية المصدرة من البطاطس بنحو

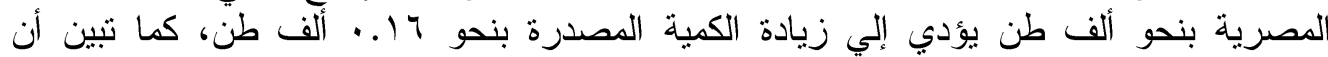

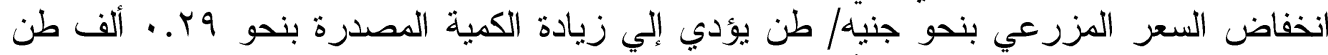

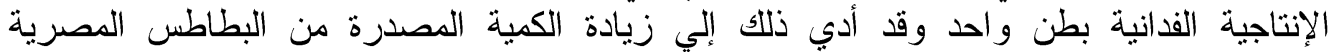

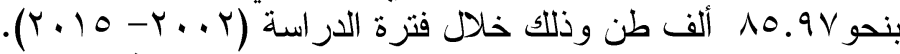

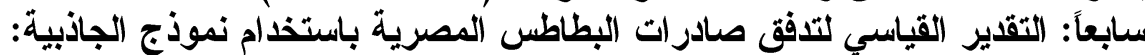

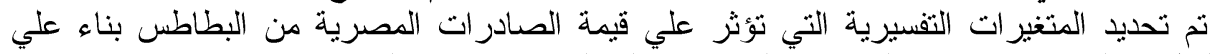

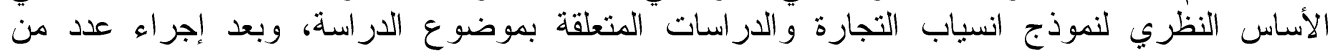

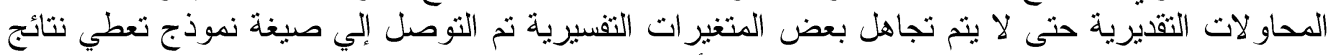

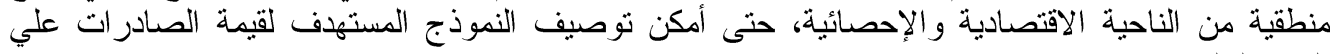
$V_{i j}=f\left(G D P_{J}+P_{J}+P R O D_{i}+I M P_{j}+D I S_{I J}+E_{I J}\right)$

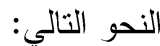

$$
\begin{array}{r}
\text { قيمة صادر ات مصر من البطاطس بالدو لار }=V_{i j} \\
\text { لالديب الفرد من الدخل القومي في الدول المستوردة بالدو لار }=\left(G D P_{J}\right)
\end{array}
$$

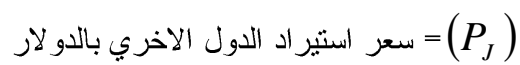

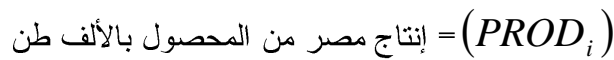$$
\text { إجمالي واردات الدول المستوردة بالطن }
$$$$
\text { المسافة الجغر افية بين مصر و الدول المستوردة بالكيلو مثر ودرد }
$$$$
\text { (POP } \left.{ }_{j}\right)
$$$$
\text { ( }
$$$$
\text { = = الاولة مصر المصدرة }
$$$$
\text { ا }
$$

Fayoum J. Agric. Res. \& Dev., Vol. 32, No.1, January, 2018 
نموذج الاتحدار المقدر لتدفق صادرات البطاطس المصرية باستخدام نموذج الجاذبية:

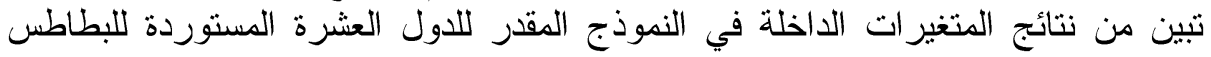

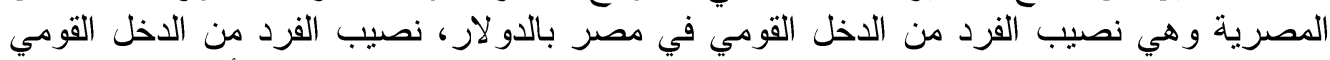

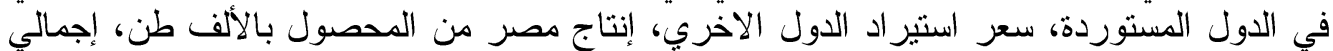

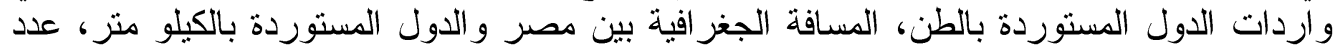

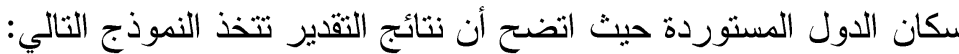
$V_{I J}=\underset{(-0.31)^{-}}{-2946}+\underset{(0.09)^{-}}{0.07} G D P_{j}+\underset{(2.37)^{5}}{18.45 P_{j}}+\underset{(1.42)^{-}}{4.36} P R O D_{i}+\underset{(6.17)^{m}}{0.02} I M P_{j}-\underset{(-6.69)^{\prime \prime \prime}}{14.01} D I S_{i j}+\underset{(8.19)^{\prime \prime \prime}}{310} .37 P O P_{j}$

$$
F=(28.02)^{* * *} \quad \stackrel{-2}{R}=0.580 \quad R^{2}=0.60
$$

يتضح من النموذج المقدر أنه لم تثبت المعنوية الإحصائية لمعامل الانحدار لكل من نصيب الكيب

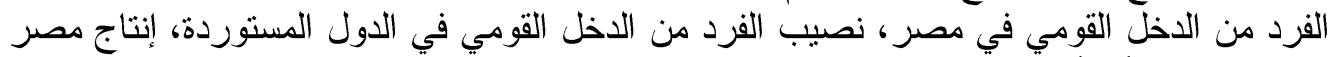

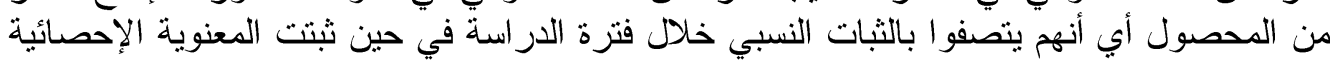

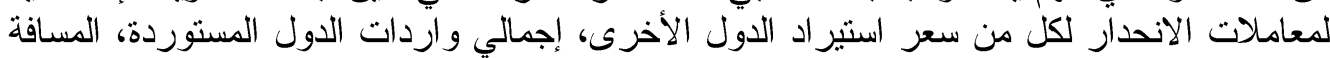

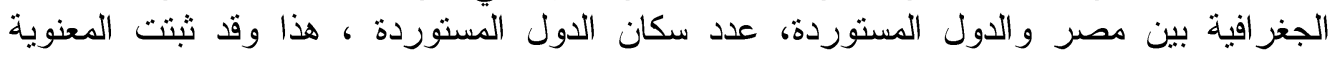

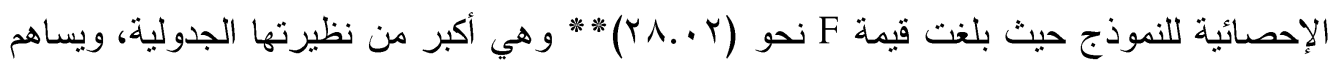

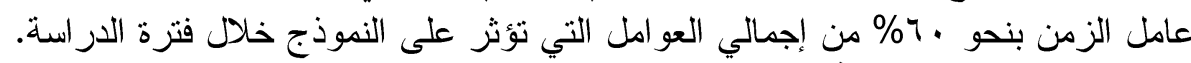

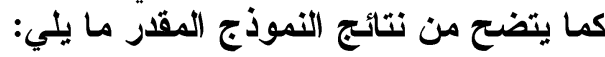

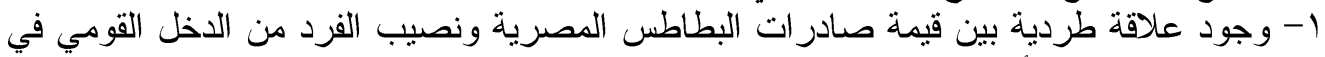

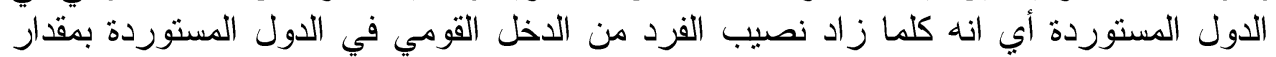

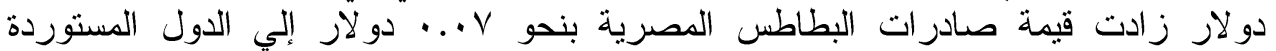

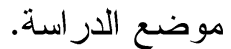

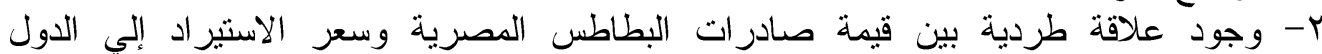

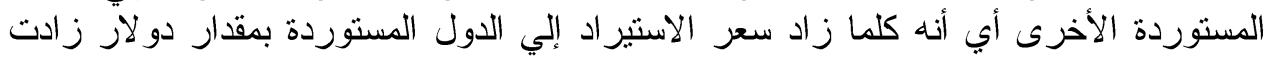

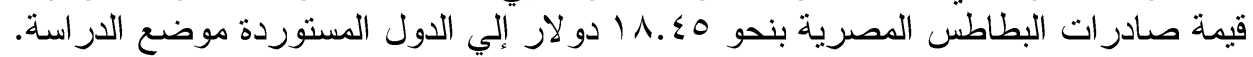

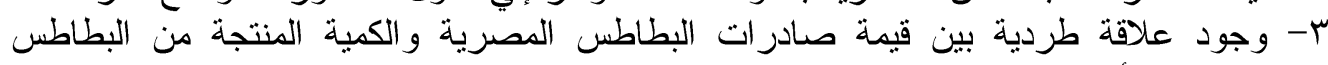

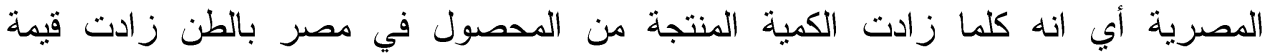

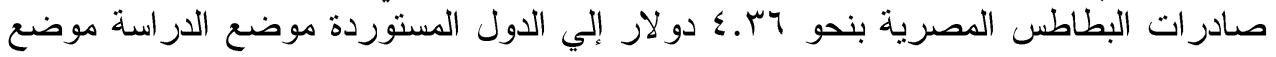
الدر اسة. ع- وجود علاقة طردية بين قيمة صادرات البطاطس المصرية وإجمالي كمية واردات الدول

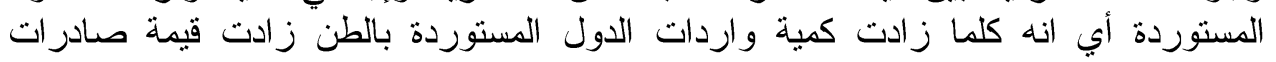

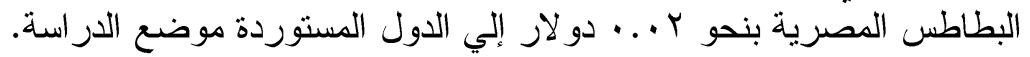

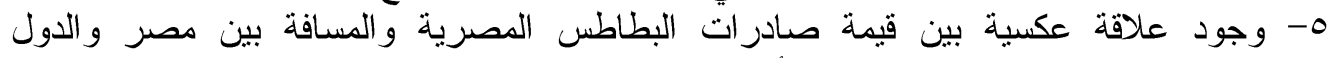

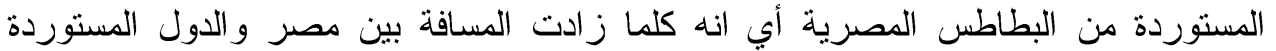

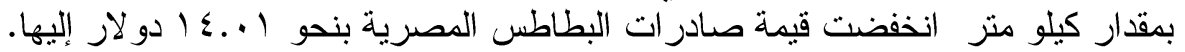

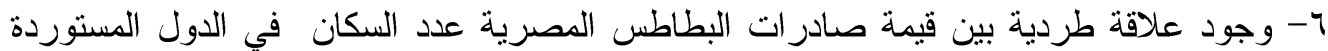

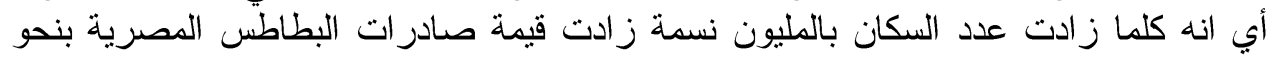

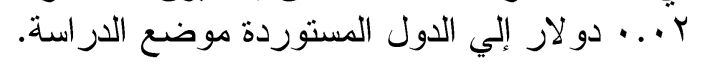

Fayoum J. Agric. Res. \& Dev., Vol. 32, No.1, January, 2018 
يستهدف البحث إبراز أهم المؤشرات التصديرية للبطاطس المصرية مع بيان معامل

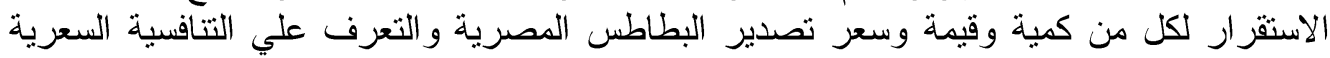

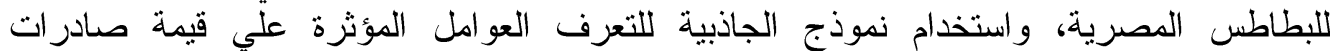

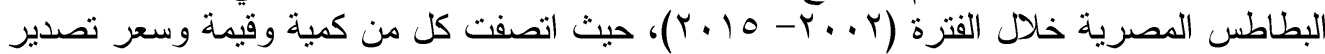

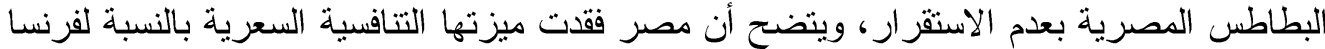

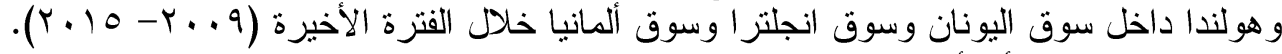

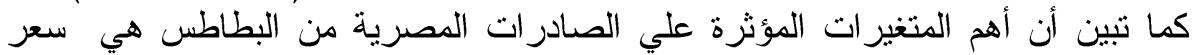

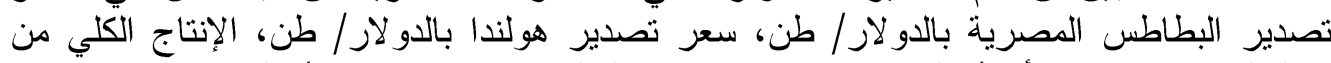

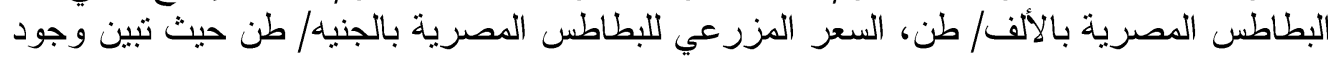

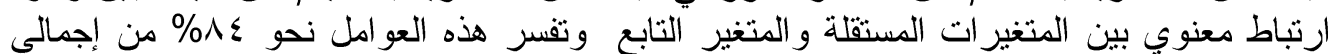

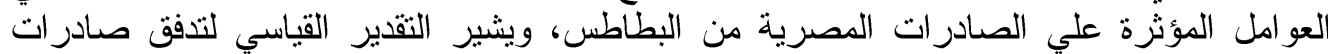

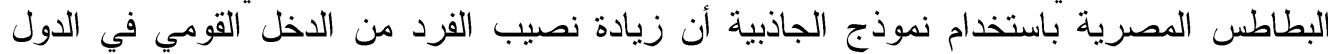

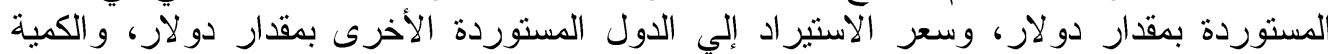

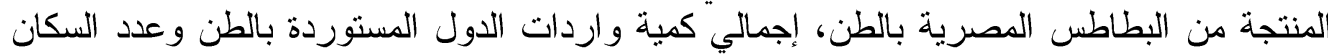

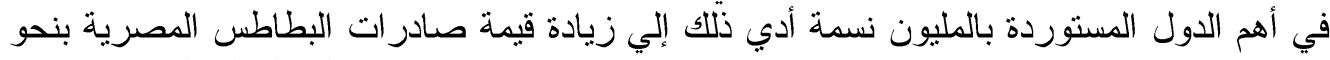

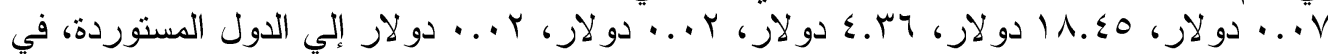

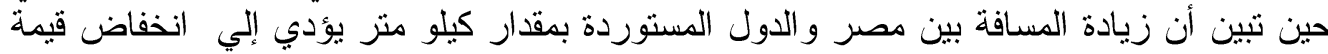

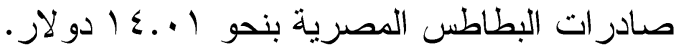

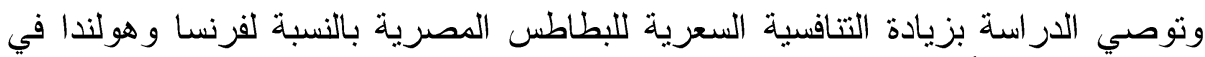

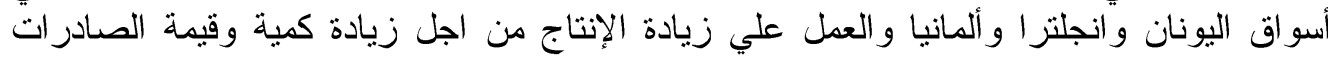

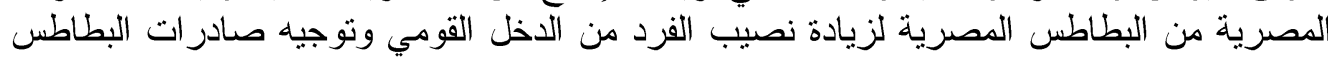

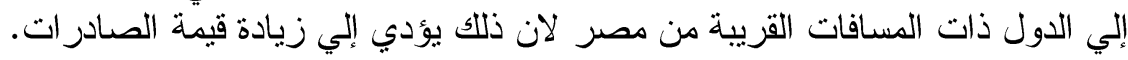

ا- وزارة الزراعة واستصلاح الأراضي، الإدارة المركزية للاقتصاد الزراعي، قطاع الثئون

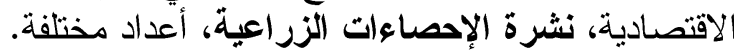

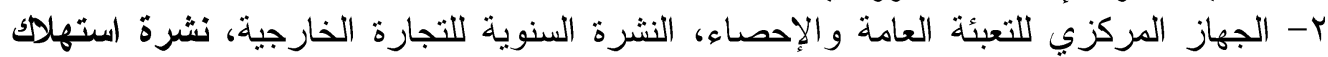

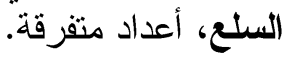

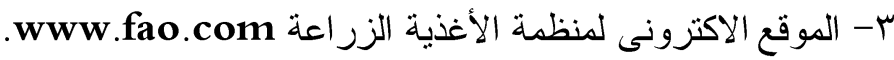

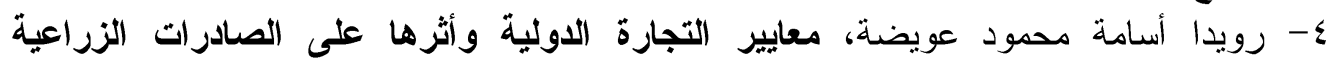

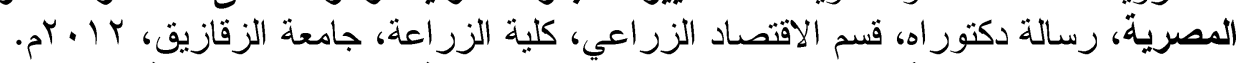

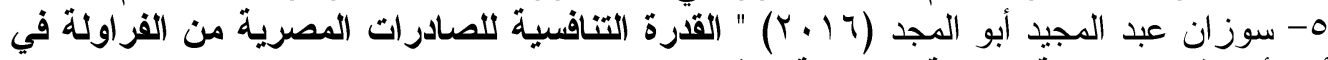

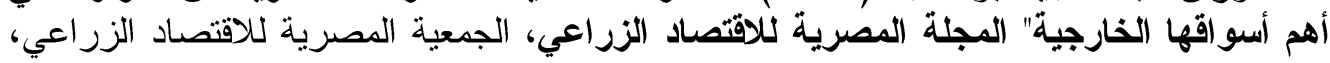

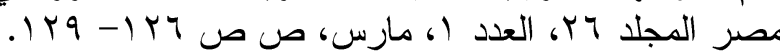

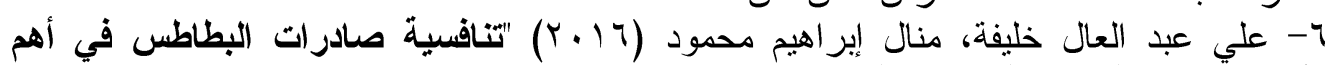

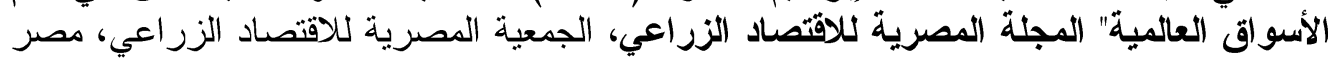

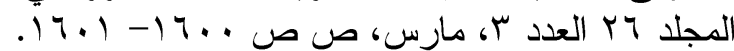

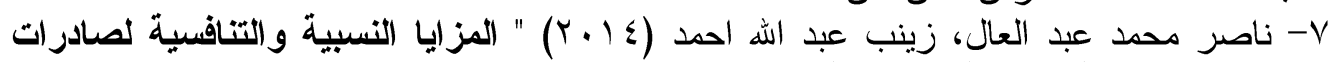

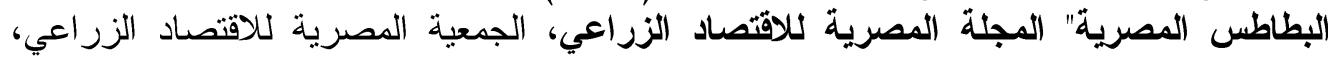

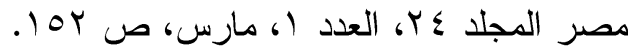

Fayoum J. Agric. Res. \& Dev., Vol. 32, No.1, January, 2018 
8- Hassanain, H. T. (2010). Gravity Trade Model: Applications on Rice and Orange. M.Sc. Thesis, Dept Agric. Econ, Fac. Agric, Zagazig University.

9-Shehata, E. A. (2011). Economic Impact for Trade between Egypt and COMESA By Using Gravity Model of Spatial Analysis, Egyptian Journal of Agricultural Economics, vol.21, No.4, Dec. 2011

10- R. Mead, R. N. Curnow and A. M. Hasted (1993) "Statistical Methods in Agriculture And Experimental Biology" Second edition Chapman \&Hall Ny. pp 213 By Using Gravity Model of Spatial Analysis, Egyptian Journal of Agricultural Economics, vol.21, No.4, Dec. 2011.

11- Ferto, Imre and Lione J. Hubbard (2002) "Revealed Combative Advantage and Competitiveness in Hungarian Agri-Food Sectors" Institute of Economics, Hungarian Academy of Sciences, Budapest, Hungary (KTK/ IE Discussion Papers 2002/ 8).

12- R. Mead, R. N. Curnow and A. M. Hasted (1993) "Statistical Methods in Agriculture And Experimental Biology" Second edition Chapman \&Hall Ny. pp 213.

\title{
ANALYSIS OF THE DETERMINANTS OF EGYPTIAN POTATO EXPORTS
}

Mohamed, H. F. and I. M. M. Jabr

Cent. Lab. for Design \& Stat. Analysis Res. ARC, Giza, Egypt

\begin{abstract}
The objective of the research is to highlight the most important export indicators of the Egyptian potato with a statement of the stability coefficient for the quantity, value and price of exporting the Egyptian potato and to identify the price competitiveness of the Egyptian potatoes and the use of the gravity model to identify the factors affecting the value of Egyptian potato exports during the period (2002 - 2015). The quantity, value and price of exporting Egyptian potatoes are characterized by unstable. It is clear that Egypt has lost its advantage of competitive price for France and the Netherlands in Greece, England and German markets during the last period (2009-2015).

IT is clear that the most important variables affecting the Egyptian exports of potatoes are the export price in dollar/ton, the export price of the Netherlands in dollar/ton, the total production of Egyptian potatoes per thousand tons, the farm price of the Egyptian potatoes in pounds/ton where there was a significant correlation between the independent variables and the dependent variable. These factors explain $84 \%$ of the total factors affecting the Egyptian exports of potatoes. The standard estimate of flow of the
\end{abstract}

Fayoum J. Agric. Res. \& Dev., Vol. 32, No.1, January, 2018 


\section{$\varepsilon q$}

Egyptian potato export using the gravity model indicates that the per capita in national income in the importing countries increases by one US $\$$ and the import price to the other imported countries by a dollar, and the quantity produced from Egyptian potatoes in tons, the total quantity of imports of importing countries in tons and the number of population in the main importing countries by one million people led to an increase in the value of Egyptian potato exports by about $0.07,18.45,4.36,0.02,0.02$ dollars to the importing countries, while increasing the distance between Egypt and the importing countries by one kilometer leads to a decrease in the value of Egyptian potato exports by about 14.01 dollar.

The study recommends increasing the competitiveness price of the Egyptian potato for France and the Netherlands in the markets of Greece, England and Germany and working on increasing production in order to increase the quantity and value of Egyptian exports of potatoes to increase per capita in the national income and direct potato exports to the countries close to Egypt because this leads to increase value of the exports.

Fayoum J. Agric. Res. \& Dev., Vol. 32, No.1, January, 2018 